\title{
Impacto de la introducción de un material didáctico interactivo en los resultados de aprendizaje en asignaturas de grado de ingenierías Impact of the introduction of an interactive didactic material on the learning results of subjects in engineering degrees
}

\author{
Hernández-Castellano, P., Marrero-Alemán, M., Paz-Hernández, R., Bordón-Pérez, P., Suárez-García, L. \\ pedro.hernandez@ulpgc.es, mariadolores.marrero@ulpgc.es, ruben.paz@ulpgc.es, pablo.bordon@ulpgc.es, luis.suarez@ulpgc.es \\ Departamento de Ingeniería Mecánica. Grupo de Innovación Educativa Ingeniería de Fabricación \\ Universidad de Las Palmas de Gran Canaria \\ Las Palmas de Gran Canaria, España
}

\begin{abstract}
Resumen- Este trabajo pretende analizar el impacto que ha tenido la introducción de un material didáctico interactivo sobre el sistema ISO GPS de especificaciones geométricas de producto. Este material ha sido desarrollado por el Grupo de Innovación Educativa Ingeniería de Fabricación de la Universidad de Las Palmas de Gran Canaria y se está usando en asignaturas de introducción a los procesos de fabricación de varios títulos de grado de ingenierías. Se plantea la necesidad de disponer de este material didáctico, la metodología seguida para su desarrollo, una breve descripción del mismo y los resultados obtenidos en su implantación. Se realiza un análisis cuantitativo y cualitativo tras su aplicación a dos asignaturas en los cursos académicos 2015/16 y 2016/17. En una de estas asignaturas se realiza una comparación con los resultados obtenidos en el curso previo a su introducción. La conclusión obtenida de este trabajo es que su impacto ha sido positivo y dispone de margen de mejora al introducir algunos cambios en la metodología de aplicación.
\end{abstract}

Palabras clave: Material didáctico interactivo, trabajo autónomo, resultados de aprendizaje

\begin{abstract}
This paper aims to analyze the impact of the introduction of an interactive didactic material on the ISO GPS system of geometric product specifications. This material has been developed by the Educational Innovation Group Ingeniería de Fabricación of the University of Las Palmas de Gran Canaria and is being used in introductory courses in the manufacturing processes of several engineering degree programs. The need to have this didactic material, the methodology followed for its development, a brief description of the same and the results obtained in its implementation is raised. A quantitative and qualitative analysis is carried out after its application to two subjects in the academic courses 2015/16 and 2016/17. In one of these subjects a comparison is made with the results obtained in the course prior to its introduction. The conclusion obtained from this work is that its impact has been positive and has room for improvement by introducing some changes in the methodology of implementation.
\end{abstract}

Keywords: Interactive didactic material, autonomous work, learning outcomes

\section{INTRODUCCIÓN}

Este trabajo se centra en la evaluación del impacto que ha tenido la introducción de un material didáctico interactivo, en los resultados de aprendizaje de asignaturas de dos títulos de grado de ingeniería. El material didáctico se centra en una introducción al Sistema ISO GPS (Geometric Product Specifications) de Tolerancias Dimensionales. Este material didáctico nace en el marco de un proyecto de innovación educativa y renovación metodológica para el diseño y fabricación de recursos didácticos que está desarrollando el Grupo de Innovación Educativa Ingeniería de Fabricación de la Universidad de Las Palmas de Gran Canaria. El objetivo básico de estos materiales es que puedan ser usados en muchas asignaturas del área de conocimiento de Ingeniería de los Procesos de Fabricación, o en algunas otras complementarias para conseguir mejorar la coordinación horizontal y transversal en los nuevos títulos de grado.

Este material didáctico surge por la identificación de dificultades de aprendizaje en este bloque de contenidos, en las asignaturas de introducción a los procesos de fabricación de varios títulos de grado. Se vio la necesidad de realizar una adecuación de los materiales tradicionales que se habían empleado, para su adaptación a las normas, nuevas o modificadas, que han surgido en los últimos años relacionadas con el Sistema ISO GPS. En este trabajo se realiza una breve descripción de la metodología aplicada en el desarrollo del material y de los diferentes bloques en que se ha estructurado, para permitir su uso como apoyo tanto para la enseñanza presencial como al trabajo autónomo del estudiante.

Se presentan los resultados obtenidos tras su introducción en dos asignaturas diferentes y en dos cursos académicos. Se realiza una comparación de esos resultados con los alcanzados en el curso previo a su implantación. Se analizan tanto aspectos cuantitativos como cualitativos para verificar el impacto que ha tenido la introducción de este material didáctico 


\section{CONTEXTO}

La necesidad de este trabajo surge tras la experiencia de un grupo de docentes del área de ingeniería de los procesos de fabricación, durante varios cursos académicos, al evaluar el tema de Tolerancias Dimensionales. Este tema se recoge dentro del bloque temático de Metrología Dimensional, en varias asignaturas equivalentes de introducción a los procesos de fabricación en varios títulos de grado. Se observó una dificultad de aprendizaje, significativa y generalizada, en la aplicación práctica de estos contenidos a unos sencillos problemas. Para su correcta resolución se requiere emplear únicamente sencillas operaciones matemáticas de sumas y restas, pero es imprescindible tener claros unos conceptos básicos mínimos que los estudiantes no estaban asimilando correctamente. Por tal motivo un porcentaje muy alto de los estudiantes, suspendían las primeras pruebas de evaluación de estos contenidos o aprobaban con unas calificaciones muy bajas que influían de forma significativa en las calificaciones globales de la asignatura.

La mayoría de estas asignaturas en los nuevos títulos de grado se encuentran en $2^{\circ}$ curso y cuentan con 4,5 ECTS, con lo que no es posible dedicar muchas sesiones presenciales a ninguno de los bloques temáticos en los que están estructuradas. En la planificación de las asignaturas, a estos contenidos se les puede dedicar entre 5 y 6 horas de clases divididas entre sesiones teóricas y sesiones de problemas realizados en las prácticas de aula. Tras analizar la situación y valorar diferentes alternativas, se decidió generar un material didáctico interactivo dirigido a estos estudiantes cuyos objetivos principales fueran los siguientes.

- Guiar a los alumnos en el aprendizaje de estos contenidos.

- Permitir su uso fuera del aula para reforzar con ello el aprendizaje autónomo.

- Adaptar los contenidos a una nueva normativa internacional de aplicación, que se encuentra actualmente en un proceso de adecuación y homogeneización.

Se desarrolló un primer material didáctico basado en una metodología que se venía usando en otros materiales didácticos generados por el Grupo de Innovación Educativa y orientados a la descripción de casos prácticos de aplicación de diferentes procesos de conformación (Hernández-Castellano et al., 2015). Se fijó inicialmente un ambicioso objetivo de abarcar, con un cierto nivel de profundidad, todos los tipos de tolerancias englobados en el sistema ISO GPS. El resultado final obtenido fue un material muy extenso en contenidos con una carga teórica importante, basada en un grupo de normas internacionales redactadas con un leguaje excesivamente técnico, que solo tendría interés para un grupo reducido de estudiantes con alto nivel de motivación. Esta estructura de material didáctico, que había demostrado buenos resultados con un tipo de contenidos más descriptivos, carecía del suficiente atractivo para ser usado de forma autónoma por los estudiantes.

De todas formas, se consideró que el trabajo desarrollado tenía un gran valor, aunque para aumentar su eficacia, debía reorientarse y dividirse en varios materiales más específicos, a la vez que era necesario replantear la estrategia para abordar este nuevo tipo de materiales didáctico.

\section{DESCRIPCIÓN}

Por todo lo expuesto anteriormente, se decidió plantear un nuevo material didáctico dirigido a una parte más específica del sistema ISO GPS, como son las tolerancias dimensionales y los sistemas de ajustes entre piezas que han de ensamblarse. Se desarrolló en el marco de un Trabajo Fin de Grado (TFG) de un estudiante del Grado en Ingeniería en Diseño Industrial y Desarrollo de Productos (GIDIDP) con un especial interés y motivación en el ámbito docente. El TFG se orientó especialmente en la búsqueda de recursos que mejorasen el atractivo del material didáctico y su grado de interactividad con el estudiante, pues los contenidos ya habían sido abordados de forma amplia en el trabajo previo. Se contó con la colaboración de un profesor de otro departamento, especializado en el diseño de sistemas de información, que conocía y había analizado con cierto nivel de detalle el trabajo anterior.

Este nuevo trabajo se inició con un amplio y detallado análisis bibliográfico sobre la elaboración y evaluación de materiales didácticos, para conocer en profundidad qué se considera un material didáctico y cuáles eran los requisitos imprescindibles a abordar. Se vio la necesidad de establecer una estructura homogénea, bien fundamentada y con un objetivo claro. Las principales características a cumplir debían ser: motivar a los estudiantes, proporcionar información adaptada, estimular y facilitar el aprendizaje, ejercitar habilidades, y guiar el proceso de enseñanza-aprendizaje, entre otras. Estas características se consiguen realizando diferentes operaciones, unas relacionadas con los contenidos y otras con las formas o aspecto de la presentación, y por supuesto, con la interactividad del medio de presentación elegido (Careaga \& González-Videgaray, 2008).

Los contenidos se inician con una introducción a la normalización y a la necesidad de su implantación. Luego se aborda en detalle el sistema de tolerancias y ajustes normalizados ISO, de acuerdo a las nuevas normas adaptadas que le son de aplicación y se resuelven, de forma detallada dos tipos de problemas clásicos en el análisis de ajustes y tolerancias normalizadas, incorporando consideraciones tanto técnicas como económicas, que justifican las decisiones adoptadas para la resolución de estos ejercicios. Se decidió, además, intentar conseguir una integración de los contenidos abordados en otros bloques temáticos de las asignaturas y con las prácticas de laboratorio programadas en las mismas. Para ello se incluye un caso práctico de ensamblaje de un conjunto de piezas, que luego los estudiantes fabrican en varias sesiones de prácticas de laboratorio, figura 1 . Se pretende con esto lograr un hilo conductor entre los diferentes bloques de contenidos de las asignaturas que reflejen claramente su interrelación.

Respecto a los aspectos formales, se ha establecido una organización homogénea de la información, tanto de texto como de otros recursos: imágenes, gráficos, planos, tablas, vídeos, y ventanas gráficas interactivas. A toda esta información se puede acceder rápida y fácilmente a través de un menú interactivo de navegación, siempre presente en la parte superior de la pantalla, y a iconos de acceso directo al sumario de los contenidos o a las páginas de ayuda y usabilidad. También se ha establecido un código de color para identificar los tipos de pieza eje o agujero, y facilitar la asimilación de los conceptos que les son de aplicación, figura 2. Se ha introducido una nomenclatura clara y homogénea, 
según las normas más recientes, que evita la ambigüedad en las definiciones de varios términos que todavía aparecen en algunas de las normas pendientes de actualizar. Se han extractado también las tablas donde se recogen los valores normalizados de amplitud y posición de las tolerancias dimensionales, para que puedan ser consultadas rápidamente en cualquier momento y aclarar las dudas que surjan durante el estudio.
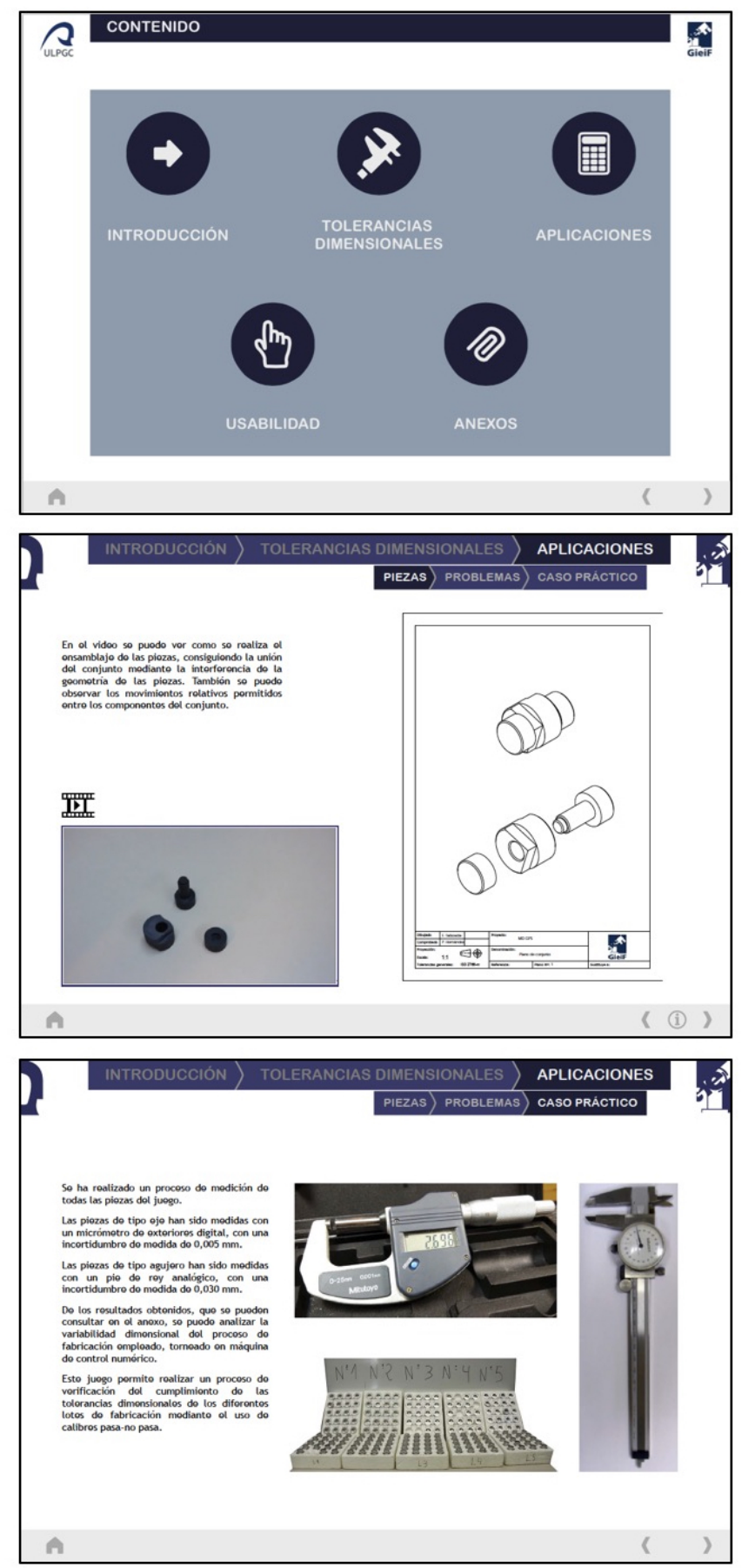

Figura 1. Páginas de ejemplo con estructura.

La metodología de utilización de este material didáctico permite, además del uso autónomo por parte del estudiante, su empleo como apoyo a las sesiones presenciales en el aula. Se ha estimado que sería necesaria una primera sesión de una hora para hacer una breve introducción a los contenidos, a la vez que se describe cómo está organizado el material, los recursos disponibles en el mismo y se dan las pautas básicas para la interacción con el documento. Se sugieren dos sesiones adicionales de entre 1,5 a 2 horas para la aplicación práctica de los contenidos. En la primera se introdujo una rápida prueba de evaluación de los conceptos básicos mediante un sencillo test, para forzar a los estudiantes a mirarse los contenidos antes de empezar con unos ejercicios de consolidación de los conceptos básicos. En la segunda de estas sesiones de aplicación práctica, se resuelven detalladamente sencillos problemas con múltiples soluciones basadas en las decisiones adoptadas, tanto de carácter técnico como económico. A este material se puede acceder libremente a través del repositorio institucional ACCEDA-ULPGC, (Hernández-Castellano et al., 2016).
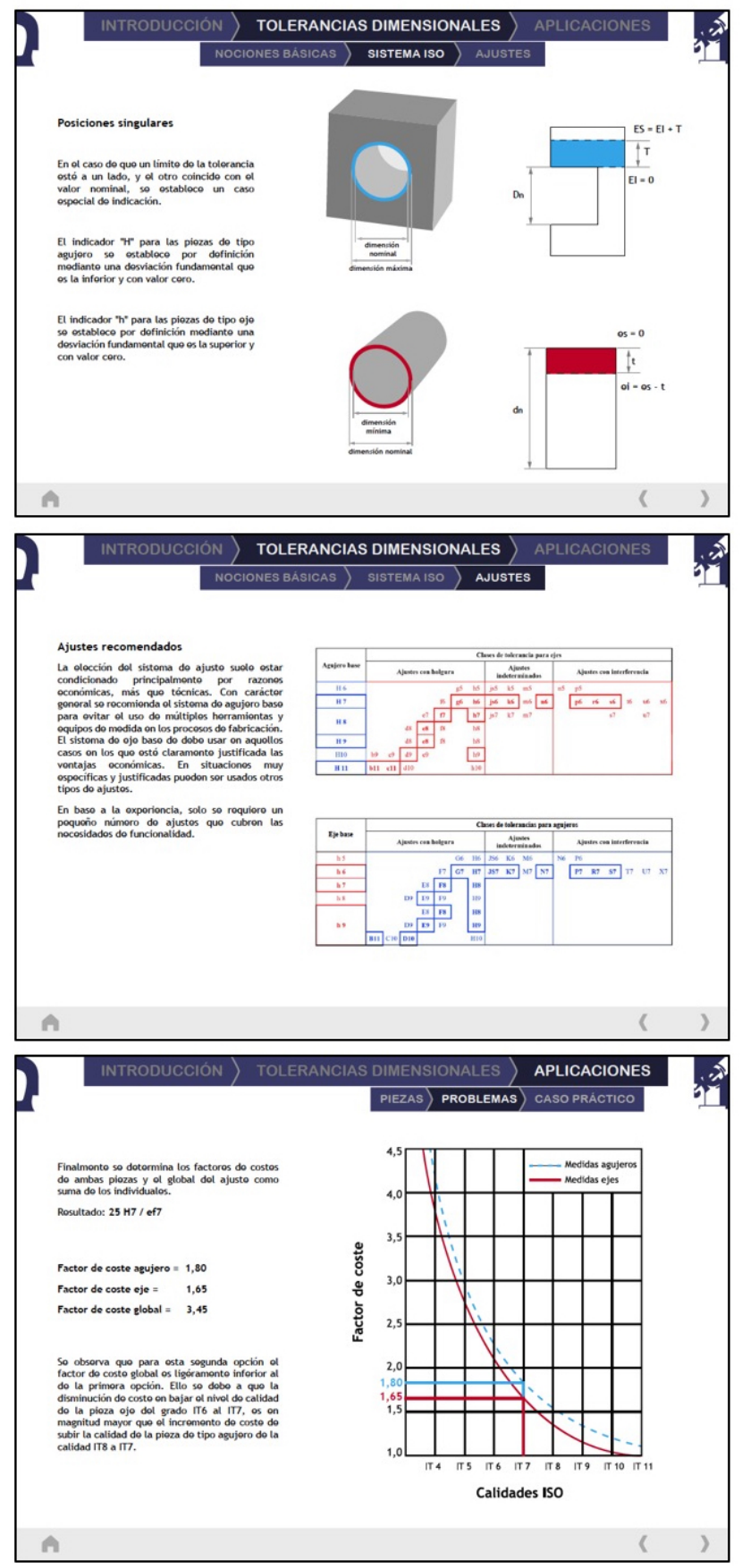

Figura 2. Aspectos formales del material didáctico. 


\section{Resultados}

La medición del impacto de la introducción de este material didáctico se ha realizado en dos asignaturas de dos grados diferentes y con diferente carga de créditos ECTS.

\section{A. Primera evaluación del impacto}

La primera experiencia se llevó a cabo en una asignatura del primer semestre del segundo curso del Grado de Ingeniería Mecánica, de 4,5 ECTS que contaba con dos grupos de teoría y 93 estudiantes matriculados en el curso 2015/16. Se decidió introducir en uno de ellos el uso de material, mientras que el otro actuaría como grupo de control y se emplearían los materiales que se habían utilizado en cursos anteriores. A su vez el grupo de ensayo se dividió en dos subgrupos, uno donde el material didáctico se usaría de forma totalmente autónoma por los estudiantes y en el otro se usaría como apoyo en clases presenciales. Se diseñó una sencilla prueba de control que consistía en diez preguntas tipo test, con cuatro respuestas posibles, y dos ejercicios para completar unas tablas con los valores que definen dos ajustes normalizados. Esta prueba, para la que se estableció un tiempo máximo de veinte minutos, permitió determinar el nivel de asimilación de los conceptos básicos requeridos para su aplicación. Fue realizada de forma voluntaria por estudiantes de ambos subgrupos de ensayo y también del grupo de control. Para evitar agravios comparativos entre ambos grupos se decidió que todos los estudiantes matriculados tuvieran acceso al material didáctico antes del examen de evaluación de estos contenidos.

Tras el análisis de los resultados de las pruebas de control se determinó que en el subgrupo de ensayo que contó con apoyo de sesiones presenciales en el aula, un 83,3\% de los estudiantes habían asimilado los conceptos analizados en un nivel suficiente, frente a un $28,6 \%$ en el subgrupo que no contó con apoyo de clases presenciales. Para el grupo de control este nivel fue alcanzado por un 58,3\%. Estos resultados claramente ponían de manifiesto que este primer impacto del material didáctico había sido positivo y significativo, y que era imprescindible unas sesiones presenciales de apoyo para una mejor asimilación de los contenidos. Se validó también este modelo de prueba de control y se decidió usar una muy similar en el examen de evaluación junto al problema de aplicación práctica descrito en el apartado anterior. Los resultados obtenidos en este examen de evaluación por los estudiantes del subgrupo de ensayo con apoyo presencial fueron del $70,6 \%$ de aprobados, con una calificación global media de 7,9 y una calificación en el problema de 8,2. Para el subgrupo de ensayo sin apoyo presencial el porcentaje bajó hasta el 35\%, aunque las calificaciones respectivas fueron de 7,3 y 8,1. Para el grupo de control los valores respectivos fueron: 71,4\%, 7,6 y 8,1.

Estos resultados muestran unas calificaciones medias relativamente altas en comparación con las obtenidas en cursos anteriores y una elevada homogeneidad de las mismas. También refuerzan que el apoyo de clases presenciales es imprescindible para una mejor asimilación de los contenidos. Este material didáctico no se planteó para su utilización de forma totalmente autónoma pues las dificultades de aprendizaje ya mencionadas, debido a lo arduo de los contenidos relacionados con una normativa árida de asimilar, no permitía pensar que se pudiera alcanzar ese objetivo. Aun con ello se decidió explorar esta opción para analizar el grado en que los contenidos recogidos en el material didáctico eran interpretados y aprendidos por los estudiantes. Claramente los resultados generales obtenidos son peores que los obtenidos en el curso anterior, aunque en algunos estudiantes concretos los resultados fueron muy similares, o incluso mejores, que los obtenidos por estudiantes del grupo que contó con apoyo presencial.

Se considera que el impacto de introducción del material didáctico en esta asignatura ha sido muy positivo, ya que además era el primer curso que se impartía de manera independiente a otros grados y además por dos jóvenes profesores, recientemente incorporados al área de conocimiento, que no habían participado directamente en la elaboración de este material. En el siguiente curso académico 2016/17 se ha aplicado la misma metodología a ambos grupos obteniendo un porcentaje de aprobados muy similar en torno al $70 \%$ con una calificación media en el problema de 8,8, y una percepción general que el nivel de asimilación de los contenidos había sido mejor que el curso anterior.

\section{B. Segunda evaluación del impacto}

La segunda experiencia de evaluación del impacto de este material didáctico fue realizada en una asignatura de tercero del Grado en Ingeniería en Diseño Industrial y Desarrollo de Productos. Esta asignatura es del segundo semestre, tiene 9 ECTS y cuenta con un único grupo de teoría y dos grupos de prácticas de aula. Se seleccionó esta asignatura puesto que se podía contar con resultados de aprendizaje comparables del curso anterior 2014/15, y el análisis se ha realizado para los cursos 2015/16 y 2016/17. Se informó a los estudiantes que el capítulo de Tolerancias Dimensionales iba a ser tratado con ayuda de este nuevo material didáctico y que requería una dedicación importante fuera del aula como trabajo autónomo para su preparación. De forma presencial se le dedicó únicamente dos sesiones de dos horas de prácticas de aula para cada grupo, en las primeras semanas del semestre. La primera sesión se dedicó a la introducción al sistema ISO GPS y a dar unas nociones básicas de la normalización de las tolerancias ISO, informándoseles que, en la siguiente sesión a realizar a los 15 días, se les someterían a una prueba de evaluación sobre los contenidos del material didáctico. Esta prueba fue muy similar a la empleada en la otra asignatura, con 10 preguntas tipo test sobre aspectos conceptuales y dos ejercicios que consistían en completar una tabla parcialmente cumplimentada con los datos de definición de un ajuste normalizado. Para esta prueba dispusieron de un tiempo máximo de 20 minutos, y su objetivo principal era el de motivar al estudiante a trabajar los contenidos antes de acudir a la siguiente sesión.

Los resultados de la prueba preliminar, para ambos cursos analizados, mostraron que la mayoría de los estudiantes habían logrado un nivel de conocimientos suficiente en la parte de aspectos conceptuales, pero igualmente la mayoría no supieron aplicarlos en los ejercicios propuestos por lo que resulta absolutamente necesario una sesión presencial para trabajar la aplicación práctica de estos contenidos. En esta prueba se pidió a los estudiantes de ambos cursos que indicaran las horas dedicadas en trabajo autónomo con el material didáctico. Se consideró que la fiabilidad de la información aportada era razonable y se observó que había una correlación media-alta entre las horas dedicadas y la calificación obtenida, siendo los estudiantes con mejores calificaciones los que más tiempo le habían dedicado al material didáctico, con un valor entre 8 y 10 horas. No se observaron diferencias significativas entre los resultados de los dos grupos de prácticas de aula en ambos 
cursos, a pesar de que uno de ellos dispuso del material didáctico una semana más, y que pudieron contar con información adicional de la prueba a través de los compañeros del otro grupo. El nivel de dificultad de las pruebas para ambos grupos fue la misma, pues éstas no diferían en contenidos, sino en el orden de las preguntas y respuestas, y en los valores numéricos de los ejercicios. Se les informó a los estudiantes de los resultados obtenidos y que serían evaluados de estos contenidos en el examen parcial de la asignatura con una prueba similar, pues se consideró muy adecuada para evaluar una competencia específica de dominio básico de las tolerancias normalizadas. A esta prueba se le sumaría un problema de tipo inverso, con una ponderación del 40\% y 60\% respectivamente.

La segunda sesión presencial se dedicó a la resolución de problemas con apoyo del material didáctico. Un primer problema, de tipo directo, fue desarrollado para definir completamente un ajuste normalizado y reforzar las nociones básicas recogidas en el material didáctico, que fue usado en el aula para aclarar algunos conceptos y consultar los valores de las tablas extraídas de la norma UNE-EN ISO 286-1. El segundo problema, de tipo inverso, podía tener múltiples soluciones y requería tomar decisiones, tanto técnicas como económicas, para justificar la solución adoptada. Se analizaron las diferentes alternativas y se establecieron las más adecuadas, atendiendo a posibles restricciones del problema. Al igual que en el caso anterior el material didáctico fue empleado como apoyo para la consolidación de conceptos básicos y para extraer la información necesaria para resolver el problema. En las sesiones, una para cada grupo, se logró que los estudiantes tuvieran un actitud más activa y participativa en el aula, sin duda motivada por tener frescos los conceptos requeridos, que permitió un mejor aprovechamiento de esta segunda sesión.

En el primer examen parcial es la prueba donde se puede evaluar con mayor fiabilidad el impacto de la introducción del material didáctico dado que es la primera ocasión en la que los estudiantes son evaluados de estos contenidos. Esta prueba de evaluación constó de 3 partes independientes, siendo una de ellas la correspondiente a Tolerancias Dimensionales, y las otras dos partes eran teóricas sobre los bloques temáticos de Metrología y de Fusión y Moldeo. Aunque se han producido unos pequeños cambios en la asignatura de un curso a otro, se considera que se puede hacer una comparación razonable de los resultados obtenidos, especialmente en los contenidos analizados. Se ha considerado que un estudiante que ha conseguido una calificación mayor o igual a 4 en este examen ha demostrado un potencial suficiente para poder aprobar con relativa facilidad la próxima prueba de evaluación. También se les pidió que indicaran cuántas horas adicionales les habían dedicado a preparar esta parte de la materia de forma autónoma y con ayuda del material didáctico, observando que los estudiantes aprobados habían dedicado entre 8 y 10 horas.

En la tabla 1, se muestran los resultados más relevantes del análisis que se ha realizado. Destaca una alta homogeneidad en el número de estudiantes presentados en el primer parcial en los diferentes cursos académicos. Se han incluido indicadores de la parte de teoría de este examen para tener una referencia global de la prueba de evaluación. De esta parte se observa que en el pasado curso académico 2015/16 se produjo una significativa bajada en porcentaje de estudiantes aprobados debido a la introducción de un nuevo sistema de evaluación independiente de los bloques temáticos. Se observa que en el curso 2016/17 se han recuperado esos indicadores, con valores similares al curso de referencia 2014/15.

Respecto a los resultados del bloque de Tolerancias Dimensionales se observa que respecto al curso de referencia también se ha producido una bajada significativa, superior al $20 \%$, en los porcentajes de aprobados que se ha mantenido constante en los siguientes cursos. Se considera que es debido a que el nivel de exigencia en la evaluación de este bloque de contenidos se ha elevado respecto al curso 2014/15, por la introducción del test para evaluación de conceptos y por una mayor rigurosidad en la evaluación del problema. Al analizar los resultados en el problema en concreto, se observa que las diferencias en porcentajes de aprobados entre el curso de referencia y los siguientes es bastante menor que respecto a los otros bloques analizados. Sí se observa una bajada muy significativa en la calificación media en los diferentes cursos debido principalmente a la mayor rigurosidad en su evaluación.

Tabla 1. Resultados del análisis de impacto.

\begin{tabular}{|c|c|c|c|}
\hline & \multicolumn{3}{|c|}{ Cursos } \\
\hline & $14 / 15$ & $15 / 16$ & $16 / 17$ \\
\hline Matriculados asignatura & 60 & 69 & 70 \\
\hline \multicolumn{4}{|c|}{ PRUEBA DE EVALUACIÓN PARCIAL } \\
\hline Presentados & 49 & 51 & 53 \\
\hline \multicolumn{4}{|c|}{ Teoría } \\
\hline Aprobados & 25 & 11 & 33 \\
\hline \% Aprobados & $51,0 \%$ & $22,2 \%$ & $62,3 \%$ \\
\hline Calificación media & 6,13 & 5,70 & 6,76 \\
\hline Estudiantes calificación $>4$ & 36 & 26 & 38 \\
\hline \% Estud. calificación $>4$ & $73,5 \%$ & $51,9 \%$ & $71,7 \%$ \\
\hline \multicolumn{4}{|c|}{ Tolerancias Dimensionales } \\
\hline Aprobados & 27 & 16 & 17 \\
\hline$\%$ Aprobados & $55,1 \%$ & $31,4 \%$ & $32,1 \%$ \\
\hline Calificación media & 7,04 & 6,71 & 6,44 \\
\hline Estudiantes calificación $>4$ & 37 & 31 & 29 \\
\hline \% Estud. calificación > 4 & $75,5 \%$ & $60,8 \%$ & $54,7 \%$ \\
\hline \multicolumn{4}{|c|}{ Problema } \\
\hline Aprobados & 16 & 13 & 12 \\
\hline$\%$ Aprobados & $32,7 \%$ & $25,5 \%$ & $22,6 \%$ \\
\hline Calificación media & 8,36 & 7,56 & 5,61 \\
\hline Estudiantes calificación $>4$ & 18 & 13 & 14 \\
\hline \% Estud. calificación $>4$ & $36,7 \%$ & $25,5 \%$ & $26,4 \%$ \\
\hline \multicolumn{4}{|c|}{ CONVOCATORIA ORDINARIA } \\
\hline \multicolumn{4}{|c|}{ Resultados acumulados en Tolerancias Dimensionales } \\
\hline Presentados & 55 & 53 & 51 \\
\hline Aprobados & 31 & 29 & 39 \\
\hline$\%$ Aprobados & $56,4 \%$ & $54,7 \%$ & $76,5 \%$ \\
\hline Calificación media & 7,18 & 7,10 & 6,69 \\
\hline
\end{tabular}

Para completar este análisis se han comparado los resultados globales acumulados obtenidos en el bloque de Tolerancias Dimensionales tras completar la evaluación de la convocatoria ordinaria. La prueba de evaluación para estos contenidos en el examen de esta convocatoria fue muy similar a la realizada en el primer parcial. Se destaca que el número de estudiantes presentados es muy similar en todos los cursos analizados. También se observan valores casi idénticos en el número de aprobados y en la calificación media en los cursos 14/15 y 15/16. En el último curso 16/17 se ha obtenido un 
porcentaje de aprobados significativamente más elevado, entorno a un $20 \%$ superior, lo que ha supuesto un traspaso de aprobados desde la convocatoria extraordinaria a la ordinaria, que también se había observado, en menor medida, en el curso anterior. Respecto a la calificación media, se ha obtenido un valor algo inferior, debido a la calificación media más baja que consiguieron los aprobados en el problema del primer parcial. Estos resultados se consideran muy positivos, teniendo en cuenta el mayor nivel de exigencia en la evaluación de este bloque de contenidos que ya se ha mencionado.

Se han introducido algunos aspectos cualitativos al análisis como que, aunque el número de estudiantes de los grupos de los diferentes cursos son casi los mismos, el grado de homogeneidad de ellos no tiene por qué ser similar. En concreto, las opiniones de varios profesores que imparten otras asignaturas en el mismo curso y a los mismos estudiantes coinciden en que el grupo menos homogéneo de los tres ha sido el correspondiente al curso 2015/16. También está la apreciación percibida por los docentes del grado relativa a que la asimilación de los contenidos, que en ocasiones no tiene una relación directa con las calificaciones obtenidas. En los cursos previos a la introducción del material didáctico, aunque los resultados no eran malos, se percibía que los estudiantes aprobaban este bloque de contenidos sin tener los conceptos lo suficientemente asimilados y ante pequeños cambios en las restricciones del problema no conseguía resolverlo de forma adecuada. Esta sensación final ha cambiado en estos dos últimos cursos, donde con carácter general se ha observado unas habilidades superiores al enfrentarse a esas mismas situaciones.

Se solicitó a los estudiantes una valoración del material didáctico a través de un foro en el aula virtual de la asignatura $\mathrm{y}$ atendiendo a diferentes criterios como facilidad de uso y manejabilidad, información gráfica y elementos multimedia, ejemplos prácticos, etc. También se les pidió que informaran de las dificultades de aprendizaje identificadas, errores encontrados, y cualquier sugerencia de mejora que consideraran oportuna. Las respuestas indicaban que les había sorprendido gratamente y que les resultaba intuitivo su uso facilitando el estudio al poder moverse con facilidad con el menú interactivo por los diferentes apartados que requerían consultar. Respecto a los contenidos les parecieron bien estructurados y presentados de forma sintética, con un formato sencillo y estructuras visuales adecuadas que les ayudaba a entender la materia.

Por todas estas razones comentadas se considera que el impacto de la introducción de este material didáctico ha sido positivo y que existe todavía un margen de mejora amplio con el que lograr mejores resultados. A ello se suma el hecho de conseguirlo con un menor número de horas presenciales, incentivando el trabajo autónomo y guiado del estudiante al disponer de un material didáctico actualizado y de utilidad para varias asignaturas.

\section{CONCLUSIONES}

Este material didáctico mejora el proceso de enseñanzaaprendizaje, potenciando las actividades de trabajo autónomo del estudiante, liberando horas presenciales en el aula para profundizar en otros contenidos.

Se ha conseguido una mejor integración entre los contenidos teóricos y prácticos de varios bloques temáticos de estas asignaturas, logrando una actitud más activa y participativa en el estudiante durante las sesiones prácticas, tanto en aula como en laboratorio.

Se ha planteado complementar este material didáctico con un módulo interactivo para la representación gráfica de los intervalos de tolerancia. También se considera necesario la introducción de unos cuestionarios de autoevaluación y un mayor número y variedad de problemas resueltos.

Se ha aprovechado la experiencia docente de miembros veteranos del Grupo de Innovación Educativa para que compañeros más jóvenes puedan desarrollar la docencia de forma más eficaz. Se ha tomado conciencia de la importancia del desarrollo de estos materiales que facilitan y homogenizan la labor docente entre grupos de diferentes titulaciones.

Los estudiantes han manifestado un alto grado de satisfacción con el material didáctico desarrollado, que se traduce en una mayor motivación y mejores resultados de aprendizaje. También en estudiantes que, en circunstancias especiales, lo han usado de forma totalmente autónoma en convocatorias extraordinarias y especiales.

La colaboración de estudiantes en el desarrollo de estos materiales ha resultado muy positiva, pues además de realizar un trabajo de gran calidad, han aportado la visión y experiencia del estudiante para conseguir una mayor facilidad en la asimilación de los contenidos.

Se considera que para impartir de forma adecuada estos contenidos se requieren unas 5 horas presenciales, distribuidas entre sesiones de teoría y sesiones prácticas de resolución de problemas. Con la información aportada por los estudiantes se estima que el tiempo necesario de dedicación no presencial para superar de forma adecuada esta parte de la asignatura está entre 15 y 20 horas.

\section{AGRADECIMIENTOS}

Se agradece al conjunto de miembros del GIE Ingeniería de Fabricación por su colaboración en este proyecto de Innovación Educativa. También se quiere agradecer a los estudiantes que han participado en el desarrollo de estos materiales didácticos por el gran trabajo realizado, y a los que han colaborado durante las experiencias de evaluación.

\section{REFERENCIAS}

Careaga, I. O., \& González-Videgaray, M. (2008). Nuevas tecnologías y educación: Diseño, desarrollo, uso y evaluación de materiales didácticos. Mexico: Trillas.

Hernández-Castellano, P. M., Marreo-Alemán, M. D., Ortega García, F., Paz Hernández, R., Socorro-Perdomo, P. P., Suárez-García, L. A., ... Hernández, C. (2015). Materiales didácticos interactivos en Ingeniería de Fabricación. II Jornadas Iberoamericanas de Innovación Educativa en el ámbito de las TICs. Recuperado a partir de http://acceda.ulpgc.es/handle/10553/16654

Hernández-Castellano, P. M., Taboada-Margalejo, E. A., Rodríguez-Perdomo, J. I., Marrero-Alemán, M. D., Ortega García, F., Socorro-Perdomo, P. P., ... SuárezGarcía, L. A. (2016). Introducción al sistema ISO GPS. Tolerancias dimensionales. Recuperado a partir de http://acceda.ulpgc.es/handle/10553/15618 